\title{
Theoretical interpretation of luminosity and spectral properties of GRB 031203
}

\author{
C.L. Bianco, ${ }^{1,2}$ M.G. Bernardini,${ }^{1,2}$ P. Chardonnet, ${ }^{1,4}$ F. Fraschetti ${ }^{5}$ R. Ruffini, ${ }^{1,2,3}$ S.-S. Xue ${ }^{1}$ \\ ${ }^{1}$ ICRANet and ICRA, Piazzale della Repubblica 10, I-65122 Pescara, Italy. \\ 2 Dip. di Fisica, Università di Roma "La Sapienza", Piazzale Aldo Moro 5, I-00185 Roma, Italy. \\ 3 ICRANet, Université de Nice Sophia Antipolis, Grand Château, BP 2135, 28, avenue de \\ Valrose, 06103 NICE CEDEX 2, France. \\ 4 Université de Savoie, LAPTH - LAPP, BP 110, F-74941 Annecy-le-Vieux Cedex, France. \\ ${ }^{5}$ Laboratoire AIM, CEA/DSM - CNRS - Université Paris Diderot, Irfu/Service \\ d'Astrophysique, F-91191 Gif sur Yvette Cédex, France. \\ E-mails: bianco@icra.it, maria.bernardini@icra.it, chardon@lapp.in2p3.fr,fraschetti@icra.it, \\ ruffini@icra.it,xue@icra.it.
}

\begin{abstract}
We show how an emission endowed with an instantaneous thermal spectrum in the comoving frame of the expanding fireshell can reproduce the time-integrated GRB observed non-thermal spectrum. An explicit example in the case of GRB 031203 is presented.
\end{abstract}

\section{Introduction}

One aim of our model (see e.g. Ref. 1 and references therein) is to derive from first principles both the luminosity in selected energy bands and the time resolved/integrated spectra of GRBs. ${ }^{2}$ The luminosity in selected energy bands is evaluated integrating over the equitemporal surfaces (EQTSs) ${ }^{3,4}$ the energy density released in the interaction of the optically thin fireshell with the CircumBurst Medium (CBM) measured in the co-moving frame, duly boosted in the observer frame. The radiation viewed in the co-moving frame of the accelerated baryonic matter is assumed to have a thermal spectrum and to be produced by the interaction of the CBM with the front of the expanding baryonic shell. ${ }^{2}$

\section{The instantaneous GRB spectra}

In Ref. 5 it is shown that, although the instantaneous spectrum in the co-moving frame of the optically thin fireshell is thermal, the shape of the final instantaneous spectrum in the laboratory frame is non-thermal. In fact, as explained in Ref. 2, the temperature of the fireshell is evolving with the co-moving time and, therefore, each single instantaneous spectrum is the result of an integration of hundreds of thermal spectra with different temperature over the corresponding EQTS. This calculation produces a non thermal instantaneous spectrum in the observer frame. ${ }^{5}$ Another distinguishing feature of the GRBs spectra which is also present in these instantaneous spectra is the hard to soft transition during the evolution of the event. $^{6-9}$ In fact the peak of the energy distributions $E_{p}$ drift monotonically to softer frequencies with time. ${ }^{5}$ This feature explains the change in the power-law low energy spectral index ${ }^{10} \alpha$ which at the beginning of the prompt emission of the burst $\left(t_{a}^{d}=2 \mathrm{~s}\right)$ is $\alpha=0.75$, and progressively decreases for later times. ${ }^{5}$ In this way the link between $E_{p}$ and $\alpha$ identified in Ref. 6 is explicitly shown. 


\section{The time-integrated GRB spectra - Application to GRB 031203}

The time-integrated observed GRB spectra show a clear power-law behavior. Within a different framework (see e.g. Ref. 11 and references therein) it has been argued that it is possible to obtain such power-law spectra from a convolution of many non power-law instantaneous spectra monotonically evolving in time. This result was recalled and applied to $\mathrm{GRBs}^{12}$ assuming for the instantaneous spectra a thermal shape with a temperature changing with time. It was shown that the integration of such energy distributions over the observation time gives a typical power-law shape possibly consistent with GRB spectra.

Our specific quantitative model is more complicated than the one considered in Ref. 12: the instantaneous spectrum here is not a black body. Each instantaneous spectrum is obtained by an integration over the corresponding EQTS: ${ }^{3,4}$ it is itself a convolution, weighted by appropriate Lorentz and Doppler factors, of $\sim 10^{6}$ thermal spectra with variable temperature. Therefore, the time-integrated spectra are not plain convolutions of thermal spectra: they are convolutions of convolutions of thermal spectra. ${ }^{2,5}$

In Fig. 1 we present the photon number spectrum $N(E)$ time-integrated over the $20 \mathrm{~s}$ of the whole duration of the prompt event of GRB 031203 observed by INTEGRAL: ${ }^{14}$ in this way we obtain a typical non-thermal power-law spectrum which results to be in good agreement with the INTEGRAL data ${ }^{5,14}$ and gives a clear evidence of the possibility that the observed GRBs spectra are originated from a thermal emission. ${ }^{5}$

\section{References}

1. R. Ruffini, M. G. Bernardini, C. L. Bianco, L. Caito, P. Chardonnet, M. G. Dainotti, F. Fraschetti, R. Guida, M. Rotondo, G. Vereshchagin, L. Vitagliano and S.-S. Xue, The blackholic energy and the canonical gamma-ray burst, in XIIth Brazilian School of Cosmology and Gravitation, eds. M. Novello and S. E. Perez Bergliaffa, American Institute of Physics Conference Series, Vol. 910 (June 2007).

2. R. Ruffini, C. L. Bianco, S.-S. Xue, P. Chardonnet, F. Fraschetti and V. Gurzadyan, International Journal of Modern Physics D 13, 843 (2004).

3. C. L. Bianco and R. Ruffini, Astrophysical Journal 605, L1 (April 2004).

4. C. L. Bianco and R. Ruffini, Astrophysical Journal 620, L23 (February 2005).

5. M. G. Bernardini, C. L. Bianco, P. Chardonnet, F. Fraschetti, R. Ruffini and S.-S. Xue, Astrophysical Journal 634, L29 (November 2005).

6. A. Crider, E. P. Liang, I. A. Smith, R. D. Preece, M. S. Briggs, G. N. Pendleton, W. S. Paciesas, D. L. Band and J. L. Matteson, Astrophysical Journal 479, p. L39 (April 1997).

7. T. Piran, Physics Reports 314, 575 (June 1999).

8. F. Frontera, L. Amati, E. Costa, J. M. Muller, E. Pian, L. Piro, P. Soffitta, M. Tavani, A. Castro-Tirado, D. Dal Fiume, M. Feroci, J. Heise, N. Masetti, L. Nicastro, M. Orlandini, E. Palazzi and R. Sari, Astrophysical Journal Supplement Series 127, 59 (March 2000).

9. G. Ghirlanda, A. Celotti and G. Ghisellini, Astronomy \& Astrophysics 393, 409 (October 2002). 


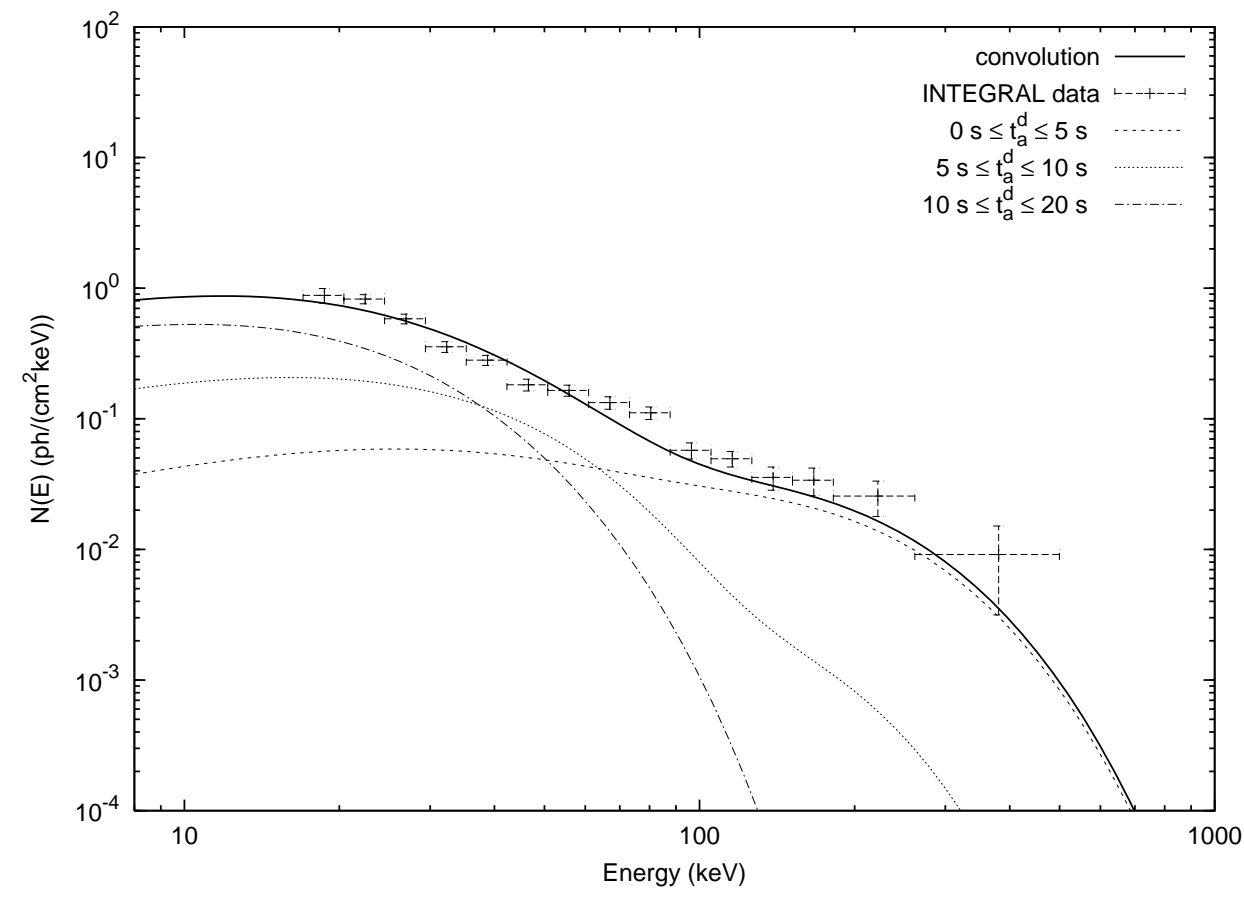

Fig. 1. Three theoretically predicted time-integrated photon number spectra $N(E)$, computed for GRB $031203,{ }^{5}$ are here represented for $0 \leq t_{a}^{d} \leq 5 \mathrm{~s}, 5 \leq t_{a}^{d} \leq 10 \mathrm{~s}$ and $10 \leq t_{a}^{d} \leq 20 \mathrm{~s}$ (dashed and dotted curves), where $t_{a}^{d}$ is the photon arrival time at the detector. ${ }^{5,13}$ The hard to soft behavior is confirmed. Moreover, the theoretically predicted time-integrated photon number spectrum $N(E)$ corresponding to the first $20 \mathrm{~s}$ of the "prompt emission" (black bold curve) is compared with the data observed by INTEGRAL. ${ }^{14}$ This curve is obtained as a convolution of 108 instantaneous spectra, which are enough to get a good agreement with the observed data. Details in Ref. 5 .

10. D. Band, J. Matteson, L. Ford, B. Schaefer, D. Palmer, B. Teegarden, T. Cline, M. Briggs, W. Paciesas, G. Pendleton, G. Fishman, C. Kouveliotou, C. Meegan, R. Wilson and P. Lestrade, Astrophysical Journal 413, 281 (August 1993).

11. L. A. Pozdniakov, I. M. Sobol and R. A. Siuniaev, Astrophysics and Space Physics Reviews 2, 189 (1983).

12. S. I. Blinnikov, A. V. Kozyreva and I. E. Panchenko, Astronomy Reports 43, 739 (November 1999).

13. R. Ruffini, C. L. Bianco, F. Fraschetti, S.-S. Xue and P. Chardonnet, Astrophysical Journal 555, L107 (July 2001).

14. S. Y. Sazonov, A. A. Lutovinov and R. A. Sunyaev, Nature 430, 646 (August 2004). 